\title{
XIII. Quantum nanoelectronics
}

The microprocessors that can be found on the market today are manufactured by etching silicon wafers with a precision as small as $14 \mathrm{~nm}$. Although this precision has dramatically improved over the years today's microprocessors are built with basically the same type of architecture developed for previous microprocessor generations. Reducing the size of elementary components even more could change the nature of the objects involved and they might no longer provide the same functionalities for which they were initially designed. In this size domain, classical physics evolves to quantum physics in many situations and, within this new physical environment, new devices have to be imagined. In this chapter we shall address some of the new phenomena and devices relying on quantum phenomena.

\section{Towards few-electron electronics}

A transistor works as a switch based on electron transport. Electron movement generates signals which are used to carry, store and manipulate information. For example, a bit can be switched from one to zero or vice versa. Several transistors properly associated together can be used to construct logical gates or memory elements. A large number of associated transistors are used to manufacture microprocessors. Shrinking transistors or memories, as it is done in the successive generations of microelectronics technology, reduces energy consumption because fewer electrons are needed to generate digital signals. This is the reason why microprocessors manufactured with smaller components are commonly used in portable computers while those with a less accurate engraving are found in desktop computers.

Electron transport requires energy. The energy is usually provided by the electrical grid or by batteries. In solid state devices such as metal oxide semiconductor field-effect transistors (MOSFET), the number of electrons required to switch or amplify a signal is of the order of 1 to 10 thousand. The efficiency of a single transistor increases as its size decreases. Reducing the distance between the source and the drain (channel) increases the operating speed. Decreasing the thickness of the oxide gate also increases the efficiency. However, below $\sim .2 \mathrm{~nm}$, tunneling effects can occur producing a leak of electrons from the gate electrode to the body and increasing the energy consumption of the MOSFET. To mitigate that effect, the silicon 
dioxide insulating material can be replaced by a material with a higher dielectric constant.

The ultimate goal would be to work with a single electron device. However, as the number of electrons and the size of the device decrease, a change in properties takes place because phenomena that were negligible at large size become dominant at tiny dimensions. Furthermore, using fewer electrons means also that the device is more sensitive to random noise from thermal background, an issue often demanding new operating conditions.

\section{Coulomb blockade}

With the possibility of working at the nanoscale, scientists and engineers have developed one-electron devices which can precisely control the flow of each electron. A fundamental principle underlying one-electron devices was discovered in 1985 by D.V. Averin and A.A. Likharev, two Russian physicists. It is the phenomenon of Coulomb blockade which means that tunneling of an electron through a metallic junction with small capacitance requires an electrostatic charging energy and thus may be inhibited at low temperatures and small applied voltages. The electrostatic potential barrier is a classical concept. Tunneling through the barrier is a quantum effect.

To elucidate this further, let's consider two metal plates separated by a thin layer of insulator such as is schematically shown in figure 98 .

1. Each metal plate is electrically neutral and has an equal number of positive charges (ions) and negative charges (electrons). The net charge of any objectisaninteger multiple of the elementary charge $e$.In other words, charge is quantized. The charge of an electron is minus the elementary charge $-e$ $\left(-1.60217662 \times 10^{-19}\right.$ coulomb $)$ and the charge of a proton is $+e(+1.60217662 \times$ $10^{-19}$ coulomb). The transfer of electrons between two objects always involves a change which is a multiple of the elementary charge $-e$.

2. The system displayed in figure 98 is a capacitor. If a positive charge is applied to one of the metal plates, an equal negative charge appears on the second metal plate. Furthermore, in a metal, the motion of electrons and the vibrations of the ions induces on the metal plates a charge which fluctuates about the mean value as a function of time allowing non-integral values. Thus although the mean charge, $Q$, on a plate is an integer multiple of the elementary charge, when $\mathrm{Q}$ is large it can be treated as a continuous variable. 


\section{Metal Insulator Metal}

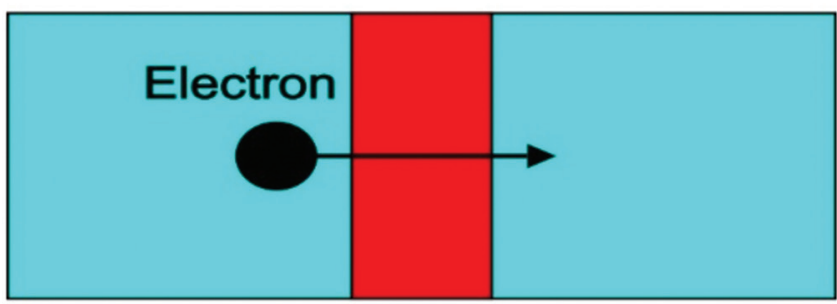

\section{Charge 0}

Metal Insulator Metal

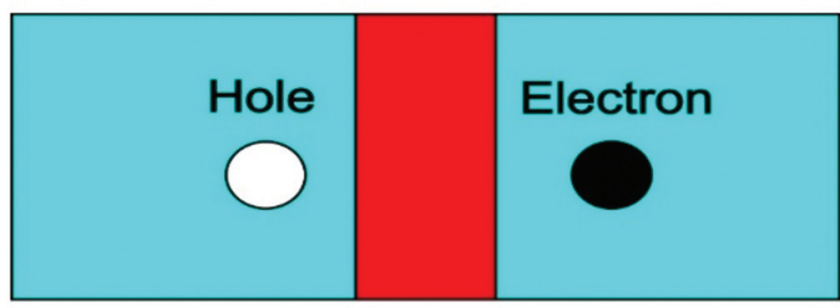

\section{$+e$ Charge $-e$}

Figure 98. Tunnel junction when the two metal electrodes are not charged. An electron cannot spontaneously go across the insulator because it costs energy. Inspired from R.Turton, The quantum dot, W.H.Freeman and Company Limited, 1995.

Consider again the arrangement of two conductors with a very thin insulating layer as displayed in figure 98, but now assume that the device is maintained at very low temperature, typically $1 \mathrm{~K}$ (one absolute degree). The low temperature ensures that the system is always in the lowest energy state and not in an excited configuration due to thermal excitation. If the insulator is thick enough, no current can pass from one conductor to the other. Such a system behaves as a capacitor. As the thickness of the insulator decreases, electron tunneling becomes possible. If we then apply a bias voltage between the two conducting electrodes an electron current can flow. Since the insulator is between two conducting electrodes, the device has both a resistance and a capacitance.

In the top of figure 98, it is assumed that there is no net charge on the electrodes. If an electron tunnels from the left side to the right side there will be one hole of charge $+e$ on the left (an electron missing) and 
one additional electron of charge $-e$ on the right. This is the configuration shown in the bottom of figure 98. Between the initial and final state there is a charge difference of $2 e$. Movement of an electron across the junction increases the energy of the system. The voltage difference $U$ induced by the tunneling is equal to $U=e / C$, where $C$ is the capacitance of the junction. If the capacitance is very small, which is the case for these nanosized systems, $U$ can be large enough to prevent further electron tunneling. A similar situation exists if tunneling occurs from the right to the left. Consequently, if no external energy is provided to the system, no additional electrons can tunnel through the junction. This is the basic idea underlying Coulomb blockade.

Due to the charge fluctuations, it is possible to momentarily have $-e / 2$ and $+e / 2$ on the left and right electrodes, respectively. In such a case, as shown in the bottom of Figure 99, an electron can move from right to left because the charge on the electrodes will just change sign but the energy of the system will remain the same. Interestingly, one electron can tunnel but not two at the same time otherwise an extra energy would be required.

Armed with this insight, we consider the more sophisticated device shown in figure 100. A small metallic dot with a size typically of the order of $100 \mathrm{~nm}$ or below, playing the role of a central electrode, is separated from external electrodes by insulator junctions. If there is no charge in the dot, the electrons cannot go across the junctions because of the Coulomb blockade mechanism. A gate, represented as a rectangle, is used to apply an external voltage. Energy can be injected into the device and the dot can be externally controlled. Applying an external voltage changes the charge on the dot. If this voltage has a value such that it alters the charge to $+e / 2$, the probability that an electron tunnels to the dot increases dramatically. Only a single electron can tunnel and the charge of the dot becomes -e/2. Now the electron in the dot can tunnel out of the dot to the second electrode. This process is known as correlated tunneling. This device allows electrons to go across the dot one by one. The flow of current is determined by the voltage applied to the gate. This works similarly to the gate of a MOSFET.

Figure 100 (1) shows the potential energy of the system as a function of the charge in each of the electrode of a tunnel junction (figure $100(3)$ ). Figure $100(2)$ shows the current as a function of the voltage applied to the junction. Coulomb blockade occurs between $-e / 2 C$ and $+e / 2 C$. The relationship between the current and the voltage does not follow Ohms law since this law would correspond to a straight line in this drawing. 


\section{Metal Insulator Metal}

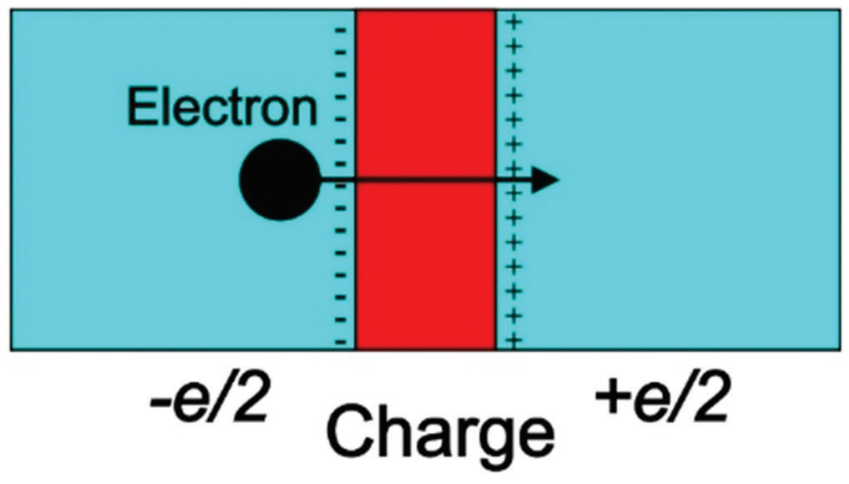

\section{Metal Insulator Metal}

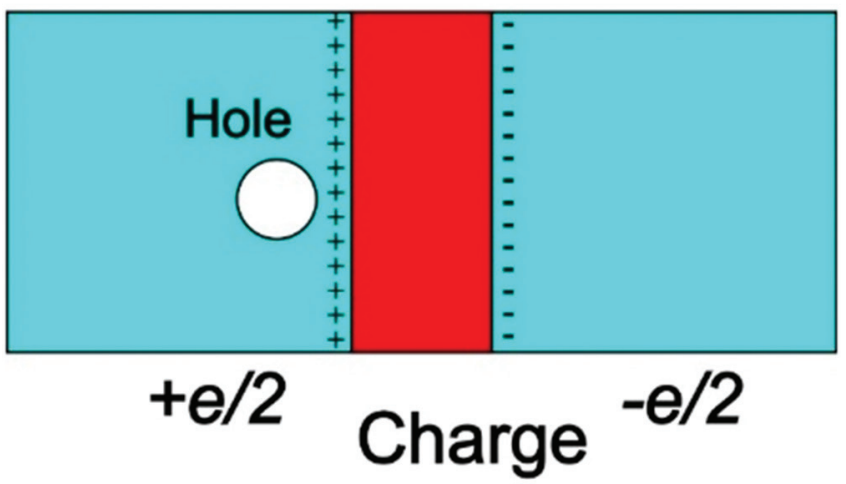

Figure 99. Tunnel junction when the two metal electrodes are charged electrically with a mean charge e/2(reflecting fluctuations). An electron can go across the insulator because it costs no energy. Inspired from R.Turton, The quantum dot, W.H.Freeman and Company Limited, 1995.

\section{The single electron transistor}

In the previous chapter we noted that transistors are three terminal devices used for signal modulation, amplification, voltage stabilization, etc. (See Figure 91). Depending on the type of transistor, an input current or voltage controls the supplied current flowing through the device. We also introduced the MOSFET (Metal Oxide Semiconductor Field Effect Transistor), a widely used unipolar transistor used in digital and analog circuits to amplify and switch signals. The CMOS (Complementary Metal-Oxide Semiconductor) technology is widely used in microelectronics for microprocessors, random access memories, digital logic circuits, etc. The word complementary means 

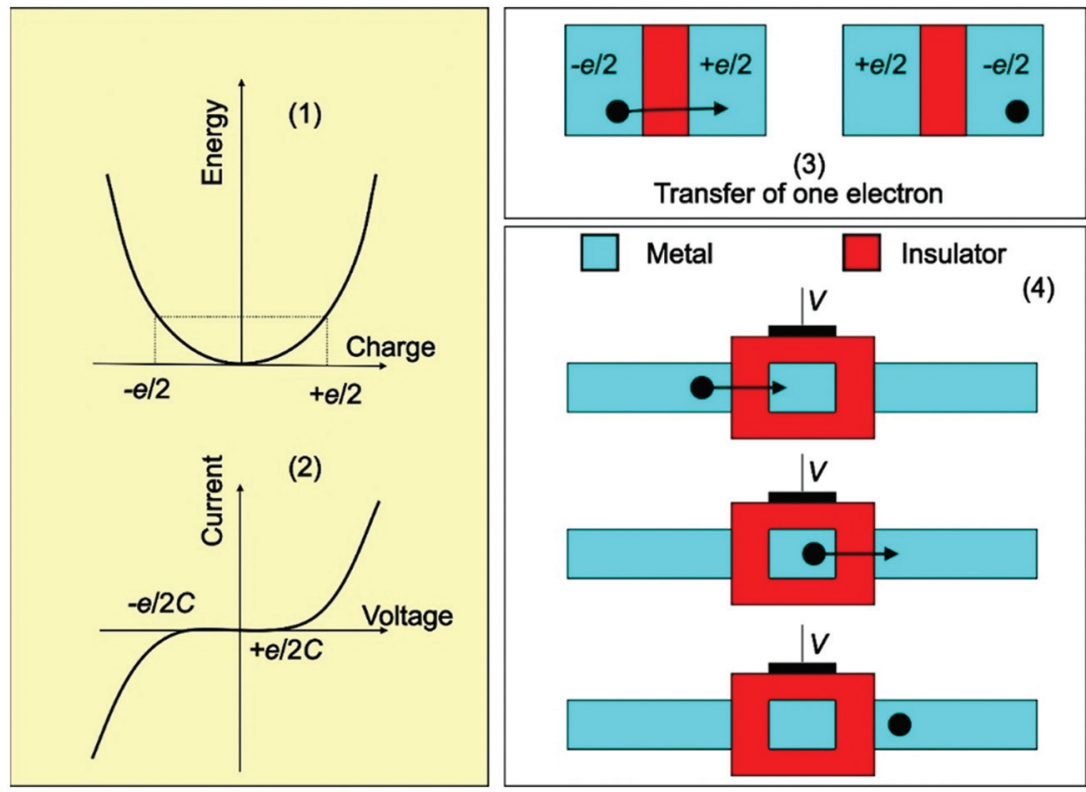

Figure 100. For the system represented in panel (3). Panel (1) shows the energy as a function of the charge of the system. Panel (2) shows the current as a function of the voltage of the system. Panel (4) shows schematically a device allowing the electrons to pass one by one (correlated tunneling). Courtesy of C.Ngô and H.Ngô, Physique des semiconducteurs, Dunod, 4th edition, 2012.

that complementary $p$-type and $n$-type MOSFET's are disposed symmetrically to provide digital functions. The advantages of the CMOS technology are that it is less sensitive to electronic noise than other technologies, and that it has a low power consumption when it is not active.

A single electron transistor (figure 101) is a switching device that has an architecture similar to that of a MOSFET but functions differently. It is a 3 -terminal device consisting of a source, a drain and a very small island of a few nanometers or less built on a semiconductor substrate. The island is electrically isolated from the drain and the source by a thin layer (typically $1 \mathrm{~nm}$ thick) of silicon dioxide playing the role of a tunnel junction. The gate is separated from the island by an insulating layer that is thick enough to prevent tunneling. The gate provides the ability to control the flow of electrons through the island by electrostatic influence. The number of electrons in the island can be precisely fixed and their flow can be completely controlled.

A single electron transistor has to be operated at very low temperature to prevent thermal background that induces thermal excitation because that would make the device useless. The operating temperature increases as 


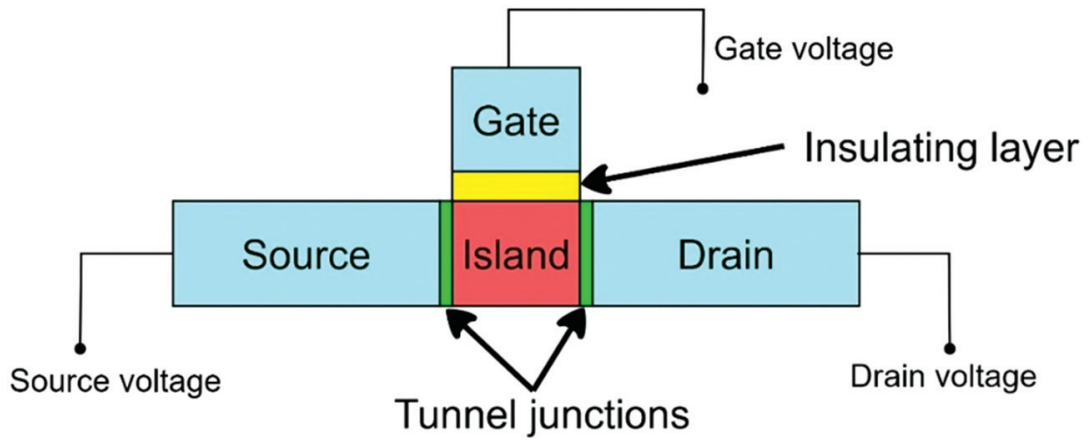

Figure 101. Principle of a single electron transistor. An island, which can be a quantum dot, is separated from the source and the drain by a very thin layer playing the role of a tunnel junction. The gate is separated from the island by a thick layer preventing electrons from tunneling through. The gate electrostatically influences the island.

the size of the island decreases because the available energy levels become more separated from each other (a quantum effect). Working at typical ambient room temperatures requires an island with a size typically of the order of one nanometer or less. The single electron transistor is an emerging technology being developed and studied in laboratories but is not yet at an industrial stage.

The function performed by a single electron transistor is different from that of a MOSFET. Consequently, different architectures must be built to treat the signal information. A wide range of research on single electron transistors built with metal, semiconductors, carbon nanotubes and molecules is underway. An example of a single electron field effect transistor manufactured by the CEA/LETI is shown in figure 102.

Applications for which single electron transistors can be used are: programmable single electron logic, single-electron spectroscopy, DCcurrent standards, electrometers, temperature standards, infrared radiation detection, etc. As an example of achievable sensitivities, a single electron transistor electrometer can detect extremely small DC currents (about $\left.10^{-20} \mathrm{~A}\right)$.

\section{Quantum dots}

A quantum dot is a nanocrystal usually made of semiconductor material in which electrons are confined in a nanoscale volume. Quantum dots were discovered by chance in the late seventies by Alexei Ekinov, a Russian physicist. He was studying the use of different kinds of alloys for microelectronics 


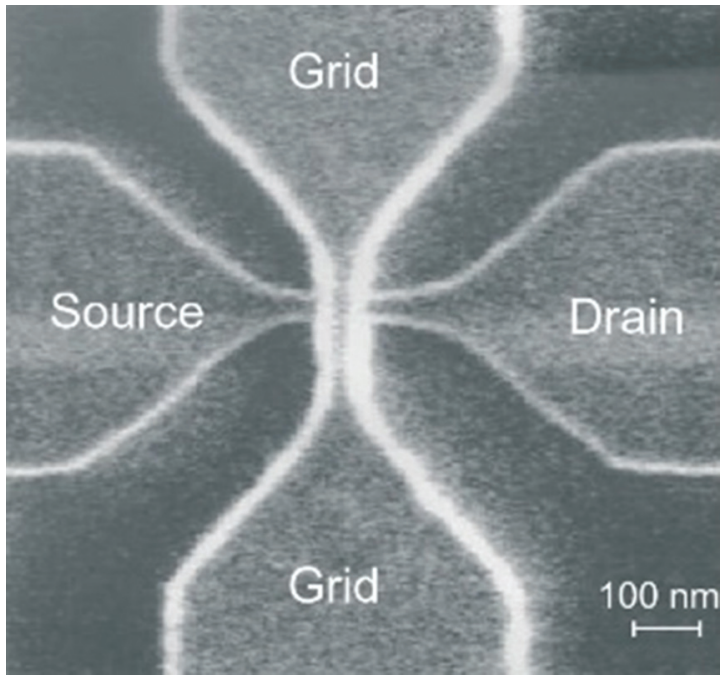

Figure 102. View of a single electron field-effect transistor manufactured in CEA/LETI. Image courtesy of CEA/LETI (France), Clefs CEA n ${ }^{\circ} 52$.

when he noticed some outstanding optical properties of nanoscale crystals of cadmium selenide, CdSe. With this discovery, the standard problem of a free particle enclosed in a nanoscale cubic box, treated in almost all texts in quantum mechanics, became reality.

In a quantum dot, the energy level separation is inversely proportional to the squares of the dimensions of the confining space of the dot. The energy difference between two energy levels is directly connected to the wavelength of electromagnetic radiation which a quantum dot can absorb and the wavelength of emission of electromagnetic radiation from a quantum dot in an excited state. Since it is possible to manufacture quantum dots with a specified size, it is possible to tailor that size to absorb or emit a particular wavelength. For electromagnetic radiation in the visible region, this means that as the size of the dot increases the color of light absorbed or emitted changes.

Figure 103 shows a schematic representation of the color of visible light emitted by excitation of quantum dots of different sizes. The color of emitted light shifts from blue to red as the size of the quantum dot increases. For a spherical quantum dot of diameter $2 \mathrm{~nm}$, light emission is in the blue region while it is the red region if the diameter is about $6 \mathrm{~nm}$.

Figure 104 shows semiconductor nanocrystals of different sizes illuminated with ultraviolet light. The absorbed color is different according to the size of the nanocrystal. One sees the wavelengths and the colors observed during illumination. 

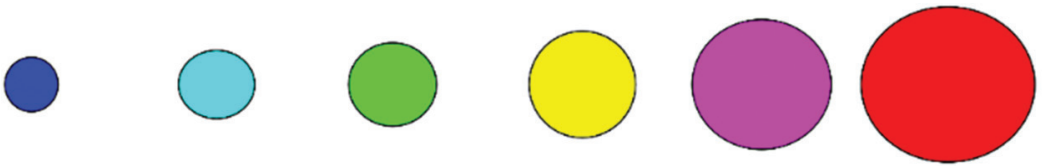

\section{Increasing wavelength}

Figure 103. Schematic illustration of the influence of the size of a quantum dot on light emission. As the size of the quantum dot increases, the color moves from blue to red.

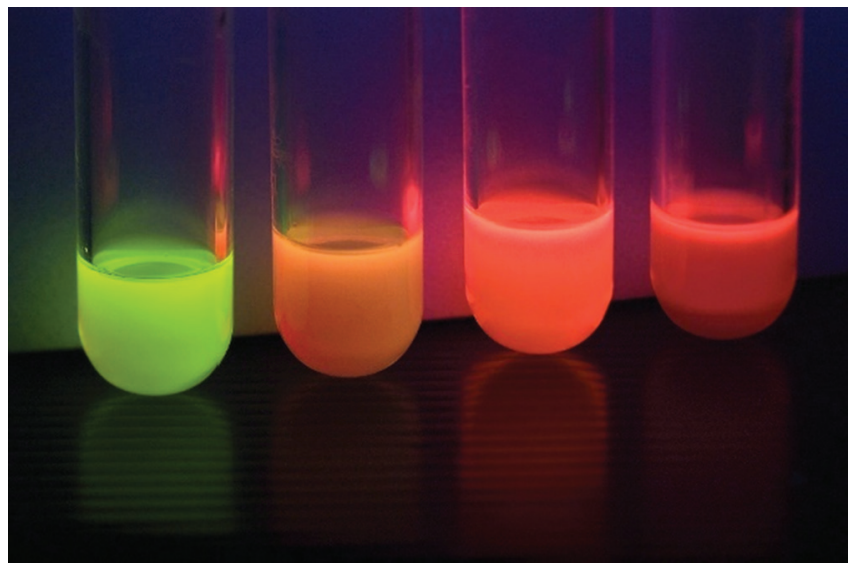

Figure 104. Semiconductor nanocrystals of different sizes illuminated with ultraviolet light. Image courtesy of CEA/LETI (France), Clef CEA n ${ }^{\circ} 52$.

Although quantum dots are usually fabricated from semiconductor materials, it is possible to manufacture a $3 \mathrm{D}$-nanoscale domain out of different materials: metals, insulators, organic substances, etc.

Figure 105 shows, in the left hand part, a quantum dot separated from metallic contacts by a tunnel junction. The top right picture in figure 105 depicts the corresponding energy levels in the potential well. The energy levels in that well can be changed continuously by changing the voltage applied to the gate. An electron located on the left and having an energy that matches that of an energy level of the quantum dot can tunnel through both barriers and go to the right. Because of Coulomb blockage, such electrons will go one by one through the quantum dot and peaks in the conductance will be observed as a function of the gate voltage (bottom right hand side in figure 105).

Because quantum dots are easily tunable they can be adapted to a wide range of applications. 

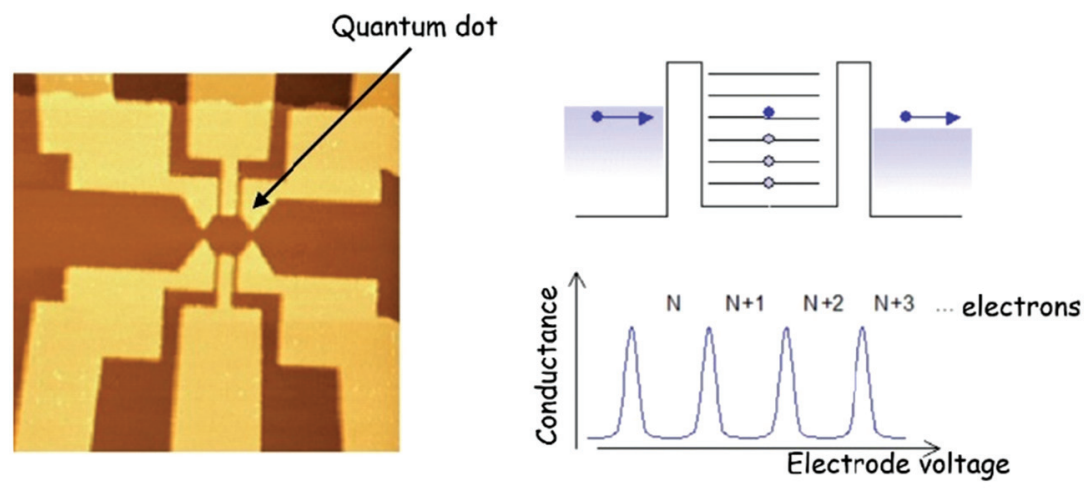

Figure 105. Quantum dot separated from the contacts by a tunnel barrier. The conductance as a function of the gate voltage exhibits peaks corresponding to the tunneling of an electron. Image courtesy of CEA/LETI (France). Clefs CEA n ${ }^{\circ} 5$.

\section{Spintronics}

An electric current in a metal or semiconductor reflects transport of charges that can be electrons (negative charges) or holes (positive charges which are actually arising from missing electrons). The propagation of a hole, an electron vacancy, is schematically illustrated in figure 106 in one dimension.

Spintronics (spin transport electronics) or spin electronics uses the intrinsic spin angular momentum of the electron together with its charge to develop new kinds of electronic devices. Associated with this spin is a magnetic moment. As a result the spin of the electron can be oriented with a magnetic or electric field.

The spin, $\mathrm{s}$, of the electron is $1 / 2 \hbar$ and two projections on the $z$ axis are possible: $s_{z}=+1 / 2 \hbar$ and $s_{z}=-1 / 2 \hbar$ often referred to as up (spin up) and down (spin down). Exploiting the spin of an electron to carry and store information is interesting because it can be done rapidly with a low energy consumption.

Spins can be spatially arranged differently depending upon the external conditions. If the electrons are free to move at ordinary temperatures, they are randomly distributed as illustrated in the left hand part of figure 107. Applying a strong magnetic field in the proper direction can align the spins as illustrated in the right hand part in figure 107.

The spins in a crystal made of non-magnetic material can be oriented at random. In magnetic materials they are aligned (see figure 108). 


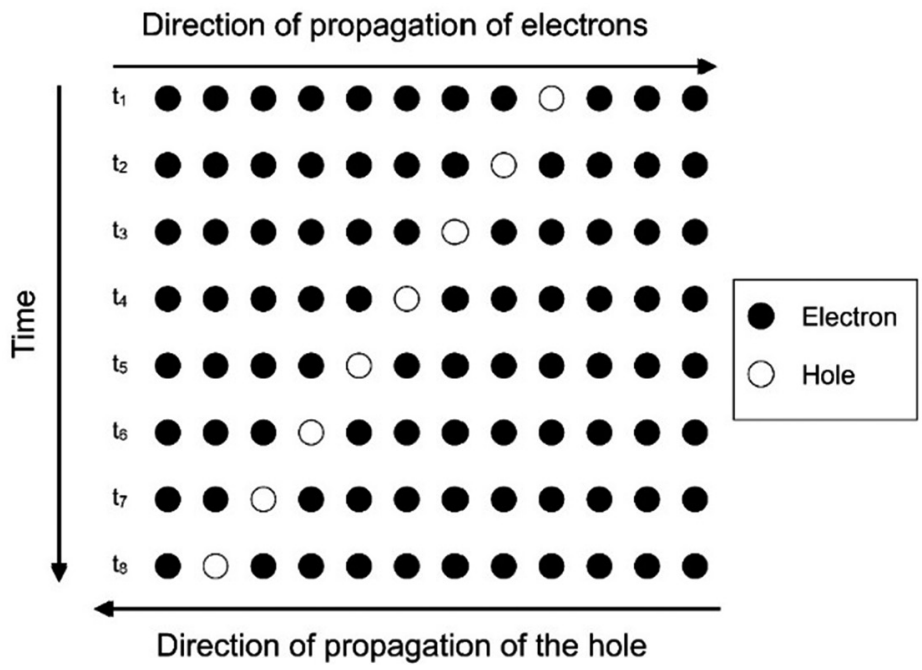

Figure 106. Propagation of a hole (site where an electron is missing) for a 1-dimensional lattice of electrons. Electrons are represented by solid black circles and the hole by a white circle. Snapshots of the lattice are shown at increasing times $\mathrm{t} 1<\mathrm{t} 2<\ldots<\mathrm{t} 8$. As electrons move to the right the hole moves to the left.

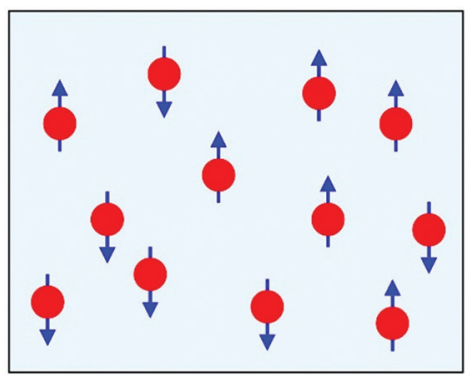

Random spins

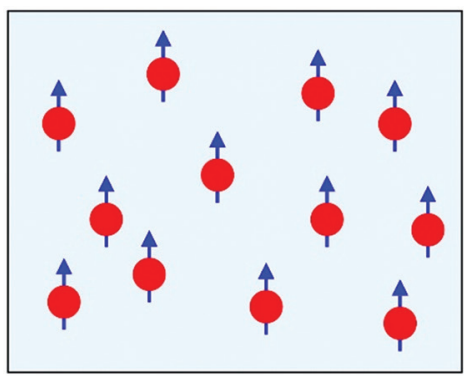

Spins aligned

Figure 107. In the left-hand part the spins are oriented at random along the same direction while, in the right-hand part, they are aligned along a strong external magnetic field. Inspired from S.D.Sarma, Spintronics, American Scientist, Vol 89, 516, 2001.

Magnetoresistance, discovered in 1856 by Lord Kelvin, is a physical effect in which a change in electrical resistance of a material occurs in response to an applied external magnetic field. The possibility to fabricate layered materials in the nanometer range led to the discovery of "giant magnetoresistance" (GMR). It is a quantum effect independently discovered in 1988 by A.Fert and his team in France, and by P.Grünberg and his team in Germany. They were awarded the 2007 Nobel prize for this discovery. 


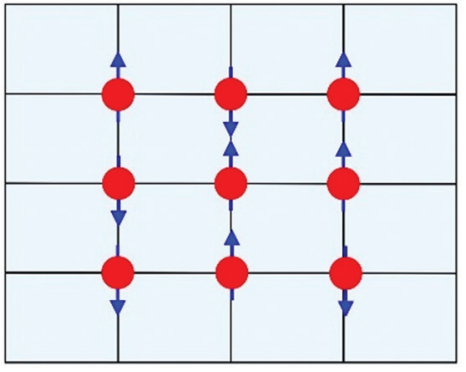

Unmagnetized

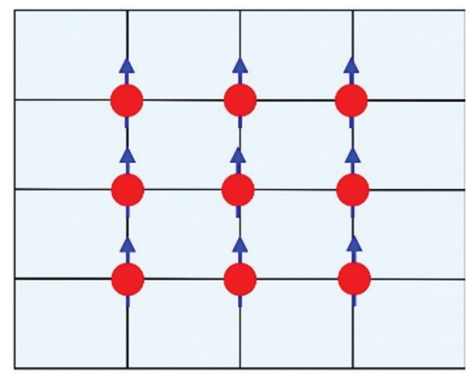

Magnetized

Figure 108. In a crystal atoms are located at specific sites. The spins will be oriented randomly in the case of an unmagnetized material or will be aligned in a magnetized material. Inspired by S.D.Sarma, Spintronics, American Scientist, Vol 89, 516, 2001.

Ferromagnetic material (Co)

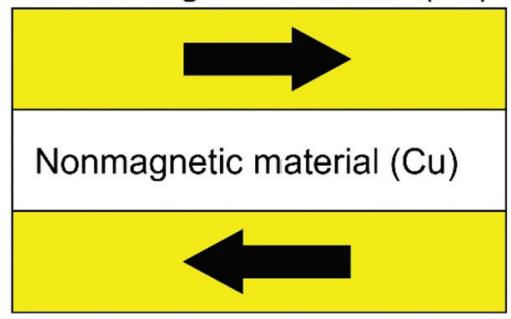

Ferromagnetic material (Co)
Ferromagnetic material (Co)

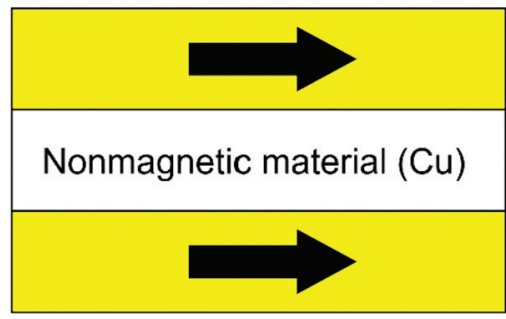

Ferromagnetic material (Co)

\section{High resistance}

\section{Low resistance}

Figure 109. Principle of giant magnetoresistance. The electrical resistance is high when the magnetizations of the ferromagnetic layers is antiparallel, while it is small when the magnetizations of these two layers are parallel.

The effect is illustrated in figure 109 using a stack of only three layers. In real experiments 10 layers or more may be involved. In the figure, a nonmagnetic layer with a thickness of the order of a nanometer is sandwiched between two ferromagnetic layers. The electrical resistance varies strongly with the relative orientation of the magnetization of these ferromagnetic layers. The electrical resistance is very high if the two magnetized layers are antiparallel (or in the case of a greater number of layers, when the ferromagnetic layers have alternating orientations of the magnetization) while is becomes very small for parallel magnetization. 
Basically, electrons with parallel spin have small scattering probabilities giving a small electrical resistance while electrons with antiparallel spins have a high probability of scattering leading to a high electric resistance. The electron mean free path, i.e., the average distance between two collisions, is of the order of 10-100 $\mathrm{nm}$. In order that the GMR mechanism appears, it must be much larger than the interlayer separation which is the case when layers with thicknesses of the order of $1 \mathrm{~nm}$ or so are involved.

Applications of the GMR include memory storage (hard disks) and magnetic field sensors. In the case of hard drives, the spin up and spin down states provide the natural basis for binary coding of information ( 0 and 1 ). Since 2000, spin-valve sensors, exploiting the GMR effect have been used in $100 \%$ of the commercial hardrives.

With spintronics, there are other possibilities than the magnetic field to control magnetization. It is possible to control magnetization with spinpolarized electric currents, electric fields or photonic fields. Spintronics devices are very small and very fast, operating on time scales of picoseconds or femtoseconds.

\section{Nanophotonics}

The US academy of sciences defines nanophotonics as "the science and engineering of light-matter interactions that take place on wavelength and sub-wavelength scales where physical, chemical or structural nature of natural or artificial nanostructured matter controls the interactions". The wavelength of visible light is in the range of about $380-780 \mathrm{~nm}$. This is far above the nanoscale domain which has been defined as the range 1-100 $\mathrm{nm}$. Nanophotonics is concerned with interactions controlled by physical or chemical properties of nanostructures or by their structure.

Nanophotonics can be divided in three main areas depending on the nanoscale confinement involved. The first area deals with the confinement of light. This includes photon localization in photonic crystals, the control of light propagation and light-matter interactions. Near field optical microscopy or photon scanning tunneling microscopy belong to this area. The second area deals with the confinement of matter into $1 \mathrm{D}, 2 \mathrm{D}$ and ${ }_{3} \mathrm{D}$ nanostructures. Nanophotonics provides the ability to control the optical behavior and the functionalities of these devices. The third area is the confinement of nanoprocesses. This deals with the nanofabrication and synthesis of devices such as nanoscale sensors, detectors and actuators or nanoscale lasers, etc. 


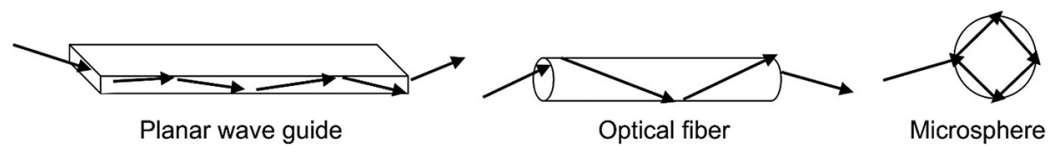

Figure 110. Schematic representation of different light confinement geometries. A planar wave guide corresponds to 1D confinement, an optical fiber to a 2D confinement and a microsphere to a 3D confinement. Inspired by A.Sharma, Nanophotonics, an overview, NSF-RISE workshop (2007).

\section{Confining and Controlling light}

Waveguides such as those illustrated in figure 110 have long been used to confine and direct light. This is with confinements operating in different space dimensions.

A waveguide usually has a structure in which a material with a high index of refraction is surrounded by a material with a low index of refraction that constitutes the cladding. Waveguides can be used in optical connections. Optical connections are interesting because light propagation in a waveguide is faster than electron transport in a conducting wire. Furthermore, the energy dissipation in the light propagation is negligible compared to energy dissipation in the electron transport (the Joule effect). However, it should be kept in mind that if light has to travel over much larger distances than electrons, the global device can be slower. In addition, the coupling between optical interconnections and conventional electrical circuits require conversion of a light signal into an electronic signal and vice versa. This requires new coupling devices that may dissipate energy and introduce time delays.

On the nanoscale, antireflection coatings and Bragg mirrors which consist of alternating layers of two different optical materials, have been used for a long time. In 1978, the first quantum well laser working at room temperature was built. The active area was only $20 \mathrm{~nm}$ thick. Quantum well lasers are now common.

\section{Photonic crystals}

Photonic crystals are artificial periodic nanostructures with spacing of the order of the optical wavelength. Before we go further it is useful to recall a few things about electrons in crystals.

A single atom has discrete levels of energy as shown in the left hand part of figure 111. These levels define the only possible values of energy that 

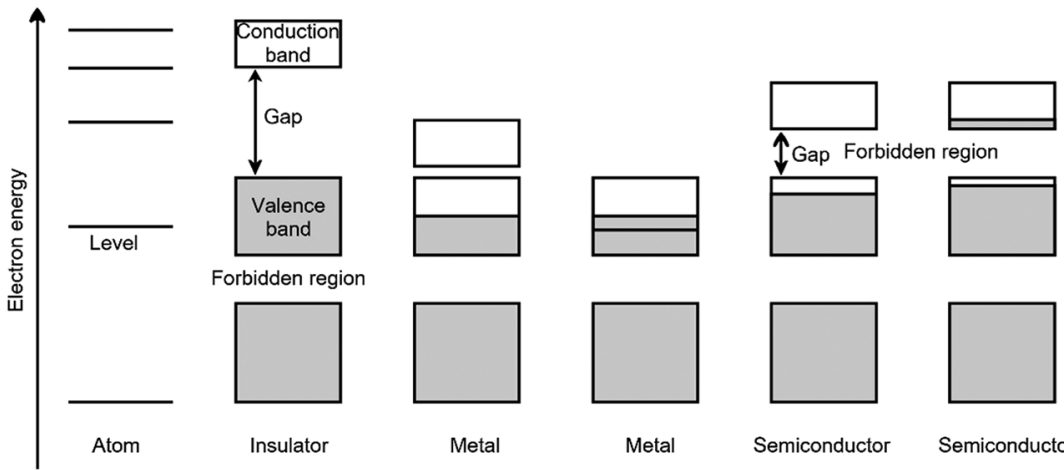

Metal

Metal

Semiconductor

Semiconductor

Figure 111. Schematic representation of the band structure of metals, insulators and semiconductors. In the left-hand side of the figure, the level structure of a single atom is indicated.

an electron in the atom can have. Other energies are forbidden. This is a direct result of the quantum mechanics that we discussed in chapter 2 . In a perfect crystal with atoms located at each site of the lattice, the energy levels transform in bands as shown in figure 111. Electrons of the crystal can only have energies belonging to a band. The regions between bands are forbidden. In the same way as the energy levels of an atom are filled from the bottom when satisfying the Pauli principle, bands are filled with the available electrons starting from the bottom. The highest energy band containing electrons is called the valence band. The lowest unoccupied band is called the conduction band. The energy difference between the top of the valence band and the bottom of the conduction band is called the band gap or energy gap.

In insulators, the valence band is completely filled with electrons and the gap is large, preventing valence electrons from being promoted into the conduction band under normal conditions. In a metal, either the valence band is incompletely filled or there is an overlap between the valence and the conduction band. In the intermediate situation, the valence band full, the conduction band empty and the energy gap small, the material is a semiconductor.

Most modern microelectronic devices use semiconductors, mostly silicon, to carry out switching and logic functions. The availability of electrons and holes in the valence and conduction bands is controlled by using dopant atoms in very small quantities. These dopant atoms possess either more or less valence electrons than silicon.

Photonic crystals, proposed almost simultaneously in 1987 by E.Yablonovitch and J.Sajeev, have some structural analogies with semiconductors or insulators. In a semiconductor, there is a periodic potential that is at the 


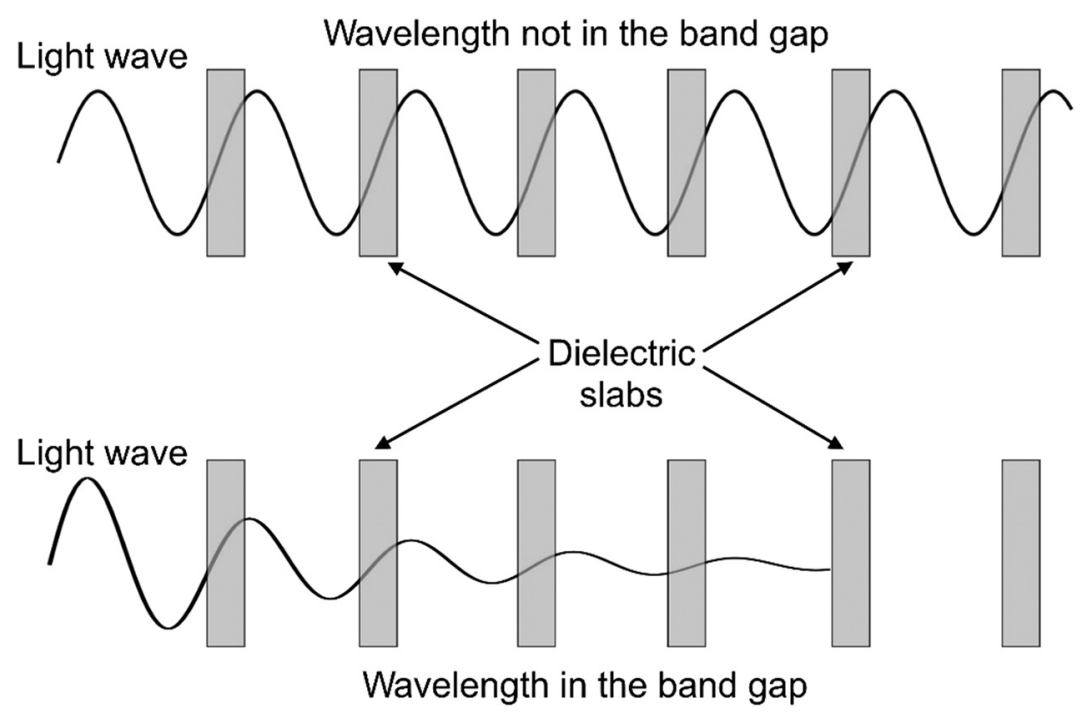

Figure 112. Basic principle of a 1D-photonic band gap material. When the wavelength is not in the band gap the incident light wave can go through the material with little attenuation. When the wavelength is in the band gap of the material, the incident light cannot go through. This comes from the fact that the reflected waves on each dielectric slab are in phase and reinforce each other.

origin of forbidden band gap for electrons. In a photonic crystal there is periodic dielectric function created by pieces of material with a different dielectric constant. This produces a periodic index of refraction that can give rise to a photonic band gap. By properly choosing the dielectric material and arrangement, it is possible to tailor the photonic bandgap. A photonic band gap serves to light in a certain wavelength range of.

Figure 112 shows the principle of a photonic band gap in a $1 \mathrm{D}$ periodic arrangement. In the $1 \mathrm{D}$-crystal, slabs of transparent dielectric material are separated from each other by air, for example. If the wavelength of the incident light is not in the band gap, light propagates through the material with very little attenuation. The incident wave is partly reflected by each slab but these waves are out of phase and cancel one another. If the wavelength is in the band gap, the reflected waves are in phase and reinforce each other. Light is blocked by the crystal.

The nice thing is that photonic crystals can be engineered on demand giving the ability to tailor the properties of the photonic band gap. In creating a photonic crystal, it is possible to play with the dimensionality of the crystal, the symmetry and topology of the lattice, the lattice parameter, the filling fraction (relative amount of refractive material), the refractive index contrast (ratio between the high dielectric constant of the material located 


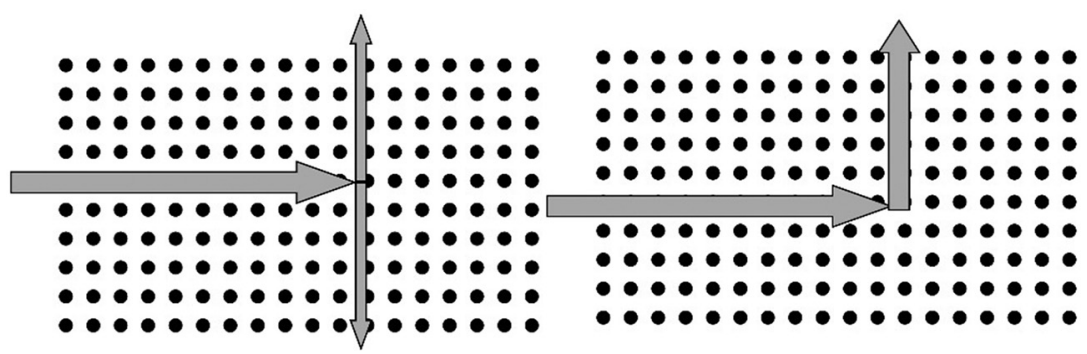

Figure 113. Schematic examples of waveguides using photonic crystals. In the left hand part, a wide-angle splitter is shown and in the right hand part a lossless sharp bend wave guide. The lattice is represented by small black dots which correspond to high dielectric material. The light wave is represented by grey arrows.

at the sites and the low dielectric constant of the remaining material) and the scalability that allows similar properties at different wavelength.

Applications of photonic crystals include supercollimators or superlenses, light confinement of (near-field and far-field microscopies), photonic integrated circuits in which photons, not electrons, carry information etc. Figure 113 shows two examples of wave guides that can be obtained with photonic band gap crystals.

Optical fibers based on photonic crystals are already commercially available. Bragg gratings are simple examples of a $1 \mathrm{D}$ photonic crystal. They have many applications, among them measurements of the structural stability in dams in order to prevent accidents.

\section{Plasmonics}

In metals, light can couple to electrons to create a wave bound to the surface of the metal that is called a surface plasmon. This provides a way to manipulate light at length scales smaller than the wavelength of the incident photons. It occurs at the interface between a dielectric such as silica glass or air, and a metal such as silver or gold. The dielectric has a positive dielectric constant while a metal has a negative dielectric constant.

A surface plasmon is a coherent electron oscillation propagating at the interface between the metal and the surrounding environment together with the electromagnetic wave associated to the incident light. In the same way as photons are the quanta associated to light, plasmons are the quanta associated to plasmon oscillations. Actually, these quanta are quasiparticles in the same way as phonons, the quanta associated with lattice vibration, 
are quasiparticles. A quantum dot, or a light emitting diode, located close to a metal surface can excite surface plasmon oscillations.

The wavelength of surface plasmons turns out to be much smaller than the wavelength of the exciting photons (typically an order of magnitude less). For example, a He-Ne laser with a wavelength of $633 \mathrm{~nm}$ can excite a surface plasmon oscillation at $73 \mathrm{~nm}$ at a silica-silver interface. By tuning the laser wavelength around the plasmon resonance, it is possible to decrease the plasmon wavelength down to the nanometer range. With such a capability it is possible to make plasmonic nanocircuits which treat light on dimensions smaller than its wavelength. Because of this, nanowires can guide light well below the diffraction limit, metal films with nanoscale holes have high optical transmissions and very thin films can be used as optical lenses.

\section{Metamaterials}

Metamaterials are engineered materials that have properties not found in nature. They are usually a complex association of components forming a composite material that can be metal-like, plastic-like or ceramic-like. The new properties are obtained from the geometrical arrangement, size, orientation, etc. of the individual elements rather than by the properties of these individual elements. In particular, these properties allow the metamaterials to block, absorb, enhance or bend electromagnetic or sound waves in a way that is not possible with conventional materials. In the domain of metamaterial, nanotechnology plays a preeminent role in constructing nanostructures that exhibit these smart properties.

In photonic crystals which have a lattice size of the order of the wavelength of light, diffraction limits the resolution. It is possible to make lattices of metamaterials with lattice constants much smaller than the wavelength of light and avoid the diffraction limit. A very active domain of research in the area of metamaterials explores negative-index of refraction materials. With such materials, negative refraction, in which light is refracted at the interface in the opposite sense to that normally expected, can be observed (figure 114).

Applications of metamaterials are possible in several areas: telecommunication, sub-diffraction imaging, sensing or invisibility cloaks. This last domain is very exciting to the general public. To be invisible, it is at least required that the refractive index of the invisible body is equal to the refractive index of air. In some wavelength regions, this can only be achieved using plasmonic metamaterials. 


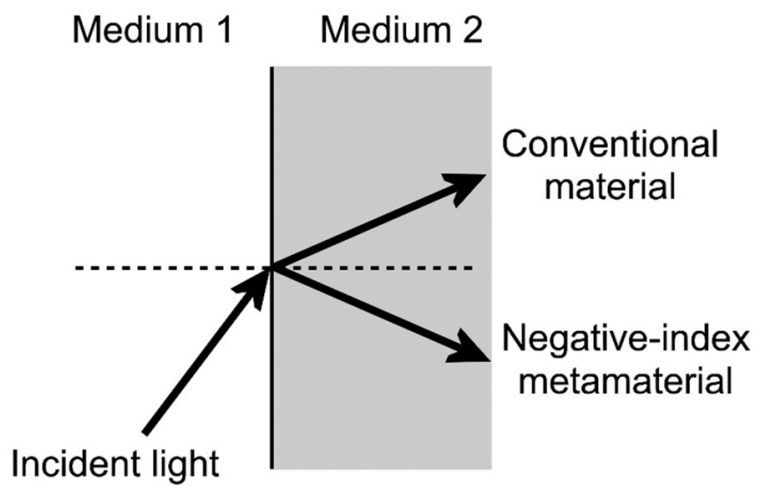

Figure 114. Diagram illustrating the difference of refraction in a medium (medium 2) having a positive-index (conventional material) or a negative-index (some metamaterials).

\section{Summary}

The appearance of new phenomena at the nanoscale paves the way to new applications. Some applications, e. g. devices based on giant magnetoresistance are already commercialized. Others such as the single electron transistor are still in the laboratory stage. Quantum nanoelectronics, which does not correspond to a simple extrapolation of conventional microelectronics, offers new opportunities to treat or store information. When the function that is possible is new or more efficient than a similar one in conventional electronics, it can be incorporated into a microdevice (nano-inside). The integration of some quantum nanoelectronic functions into conventional devices will probably be the future evolution of microelectronics:

In the more distant future, the further evolution of quantum nanoelectronics may make quantum computing available at large scale. Quantum computers are for the moment at the research stage. They use Qbits (quantum bits) which are not limited to two states as it is the case with bits used in conventional computers. Quantum cryptography, which is an application of quantum entanglement, a non-local property of quantum objects, could be used to make unbreakable cryptosystems, also an interesting application. 